\title{
Rapid ethnobotanical appraisal on Bugun, Sartang and Monpa communities of West Kameng and Tawang Sectors of Arunachal Himalayan Region, India
}

\author{
Deki Tshering ${ }^{1}$, Sanjib Das², Jambey Tsering², Pallabi K. Hui ${ }^{2}$ and Hui Tag ${ }^{1}$ \\ ${ }^{1}$ Plant Systematic \& Ethnobotanical Research Laboratory, Department of Botany, Rajiv Gandhi University, \\ Rono Hills, Doimukh-791112, Arunachal Pradesh, India \\ ${ }^{2}$ Department of Biotechnology, National Institute of Technology (NIT), Yupia-791112, Papum Pare, \\ Arunachal Pradesh, India \\ ${ }^{3}$ Orchid Research Centre, Tippi, Balukpong - 790114, West Kameng District, Arunachal Pradesh, India \\ Corresponding Author, E-mail: huitag2008rgu@gmail.com
}

[Received 21.11.2018; Revised 22.12.2018; Accepted 27.12.2018; Published 31.12.2018]

\begin{abstract}
Present paper discusses the outcome of rapid ethnobotanical appraisal made on three culturally distinct and heterogeneous communities namely, the Bugun, Sartang and Monpa of West Kameng and Tawang sectors of Arunachal Himalayan Region of India. The ethnobotanical resources of the local communities were documented using semi-structured questionnaire and focused group discussion session conducted in 6 selected villages of rural and semi-urban biocultural landscape. Investigation have revealed 77 ethnobotanically important species of which $71 \%$ are harvested from the wild and only while $29 \%$ are harvested from the cultivated sources. The most frequently used species prioritized has the potential to boost economy and ensuring rural livelihood security in the region.
\end{abstract}

Key words: Ethnobotany, Bugun, Sartang, Monpa, West Kameng, Tawang, Arunachal Pradesh

\section{INTRODUCTION}

The State of Arunachal Pradesh is a part of the Eastern Himalaya, whch is located within IUCN recognized Himalaya Biodiversity Hotspot, rich in ethnobotanical and cultural diversity (Mazhar et al. 2007). Arunachal Pradesh is a home to 26 major tribes and 110 subtribes with unique and rich indigenous knowledge system related to utilization of plant resources of their local biocultural landscape for sustenance of livelihood (Srivastava et al. 2010). West Kameng and Tawang Districts of Arunachal Pradesh is rich in forest, biodiversity and ethnobotanical heritages which are nurtured by the indigenous communities, namely, Monpa, Bugun, Sartang, Sherdukpen, Aka and Miji in their traditional ethnocultural landscape since time immemorial. The Monpa is the largest ethnic group with a population of around 0.8 lakh souls (Anonymous 2011). Perusal of literature have revealed that no such ethnobotanical information are available to date on the target lesser known local communities namely, Bugun and Sartang tribes of West Kameng District, whereas few literature evidences are available on Monpa communities reported by Tag et al. (2014) and Tsering et al. (2016, 2017). However, these literatures on Monpa have suggested that further cross-cultural ethnobotanical studies focused on food, medicinal and other livelihood plants of culturally distinct heterogeneous local communities of the region is essential which would throw more light on 
diversity, distribution and economic potential of the important ethnobotanical heritage of the region. Therefore, present study has been conducted with aims to document ethnobotanical heritage and associated traditional knowledge of three culturally and dialectically distinct local communities, namely, Bugun, Sartang and Monpa of West Kameng and Tawang Districts of Arunachal Pradesh, India and to prioritize the economically and commercially viable ethnobotanical resources of the region for ensuring rural livelihood security.

\section{MATERIALS AND METHODS}

\section{Study area and Local communities}

The study sites, Tawang and West Kameng districts of Arunachal Pradesh represents 11.35 $\%$ of the total geographical area of the state $(83,743 \mathrm{sq} \mathrm{km})$. It is located between geographical coordinates of $26^{\circ} 55^{\prime} \mathrm{N}$ to $27^{\circ} 522 \mathrm{~N}$ latitudes and $91^{\circ} 322 \mathrm{E}$ to $92^{\circ} 55^{\prime}$ to E longitudes (Figure 1) covering an area of 9,507 sq km with elevations ranging from $200 \mathrm{~m}$ in the foothill region, which gradually ascends to about $7,750 \mathrm{~m}$ above mean sea level. These two districts are bounded by China (Tibet) in the North and Bhutan in the West, and East Kameng in the Eastern side (Anonymous 2011). The vegetation are tropical semi-evergreen forest (300 $800 \mathrm{~m})$, sub-tropical broad-leaved forest $(800-1800)$, pine forest $(1200-2400 \mathrm{~m})$, temperate

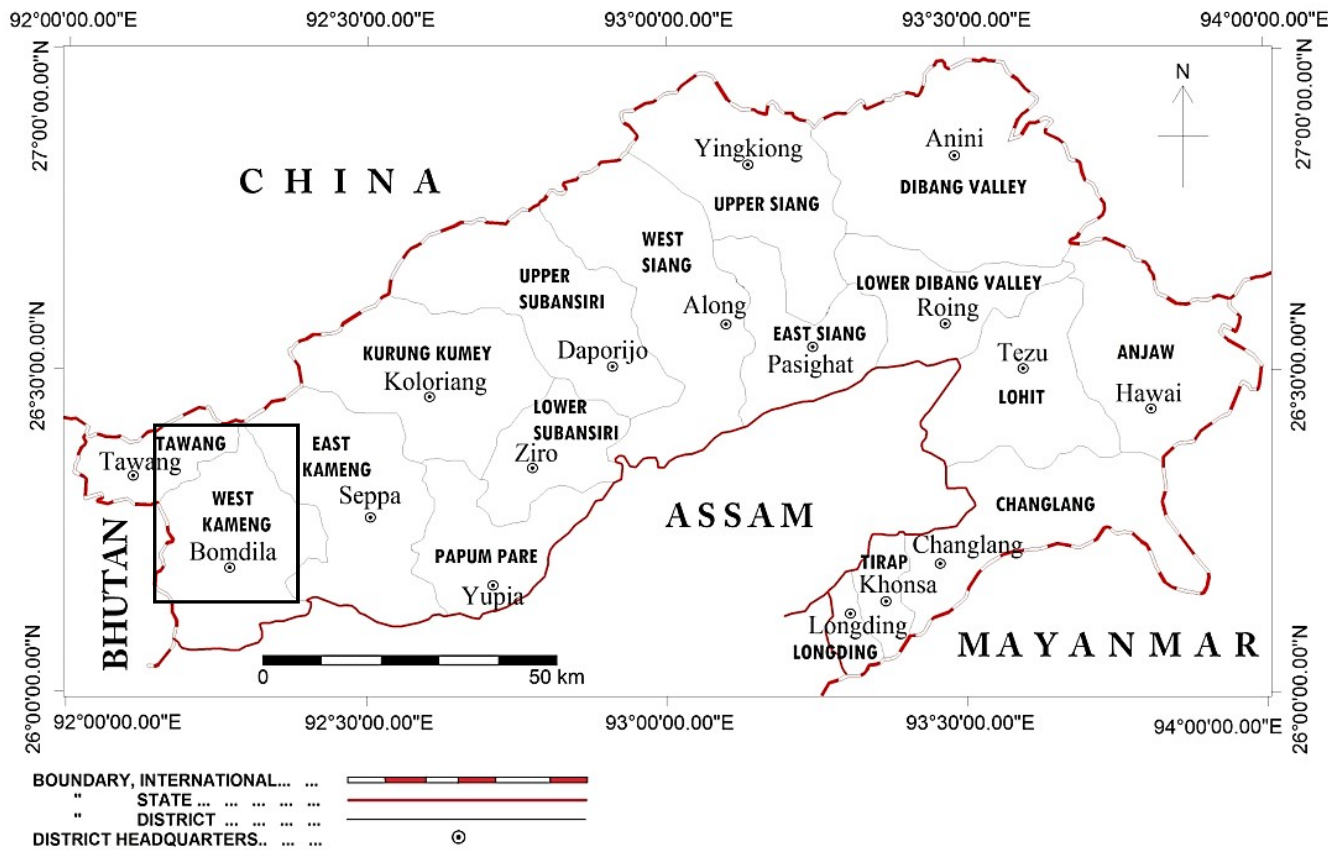

Figure 1. Map of Arunachal Pradesh showing encircled study area in West Kameng and Tawang District of Arunachal Pradesh

broad-leaved forest $(1800-2800 \mathrm{~m})$, temperate coniferous forest $(2800-3500 \mathrm{~m})$ and alpine forest above $3500 \mathrm{~m}$ (Tag et al. 2014). The climate in summer is pleasant and extreme chilling during the winter, and receives rainfall from both Northeast and Southwest Monsoon during May to September. Tawang is mainly inhabited by Monpa whereas West Kameng District is inhabited by Bugun, Sartang, Monpa, Sherdukpen, Sajolang, and Hrusso. Among them, the Monpa is the largest community in terms of population and area of occupancy, whereas, Bugun and Sartang are lesser known tribes having very less number 
of population (Norbu 2008). The target communities, namely Bugun (Khowa) is culturally distinct from rest of their counterpart tribes which has a population of around 3000 souls and they follow indigenous religion which are mainly found in Sinchung Circle of West Kameng District. Pham kho is the community festival celebrated every calendar year to worship the nature. Sartang is another culturally distinct tribe previously known as Bhut Monpa is scattered over Nafra Circle of West Kameng District and their population is around 5000 souls who follow both Buddhism and animistic religious practices. Monpa is the major tribal community scattering over Tawang and West Kameng Districts of Arunachal with total population of around 80,000 souls who mostly follow Buddhism and Lamaism. Losar is their major community festival dedicated to worshiping the nature for bountiful harvest (Tsering $\&$ Tag 2015).

\section{Survey Method}

The rapid ethnobotanical field survey was conducted in 06 villages of West Kameng and Tawang Districts of Arunachal Pradesh, namely, Lumla (Monpa tribe) under Lumla circle of Twang District, Darbu and Salari (Sartang tribe) under Dirang circle of West Kameng District, Singchung, Ramayan and Wanghoo (Bugun tribe) under Singchung circle of West Kameng District of Arunachal Pradesh during June 2017 to October 2018 following the method suggested by Martin (2008). The traditional uses of ethnobotanical resources were documented using semi-structured questionnaire and a focused group discussion session conducted in 6 selected villages of rural and semi-urban biocultural landscape inhabited by three culturally distinct heterogeneous local communities namely, Bugun, Sartang and Monpa. In all, 30 informants of age between $20-80$ years were selected for the interview of which 13 were female and 17 male members. Prior Informed Consent (PIC) were obtained from the knowledgeable local informants prior to extensive field work. Translation of local nomenclature of plants and associated traditional knowledge were assisted by local knowledge holders of the three target communities. The ethnobotanical uses, local names, parts used, mode of use, habits, and habitats and altitudinal range of each plant species were recorded in Field Note Book. Botanical name of each species were identified by consultation of standard floristic literatures such as Flora of Assam (Kanjilal et al. 1934 -1940), Flora of British India (Hooker 1875 - 1897), e-Flora of China, and e-Herbarium of Kew. The accepted names were verified in the website www.theplantlist.org hosted by RBG Kew and Missouri Botanical Garden, St. Louis, USA. The voucher specimens were prepared following the method suggested by Jain \& Rao (1977) which were deposited in the Herbarium of Arunachal University (HAU), Department of Botany, Rajiv Gandhi University, Rono Hills, Doimukh, Arunachal Pradesh for future reference.

\section{RESULT \& DISCUSSION}

Rapid appraisal has revealed 77 species of ethnobotanical resources belonging to 69 genera and 45 plant families which is presented in Table 1. Among these, herbs represent highest number of $34(45 \%)$ species which is followed by trees with 20 (36\%) species, shrubs 13 (17\%), climbers 7 (9\%), creepers 2 (3\%) (Figure 2).

\section{Diverse uses of ethnobotanical species}

Of the total 77 species reported, majority of the 49 (48\%) ethnobotanical species are used for ensuring rural food security, while $23(22 \%)$ species are used as medicinal agents to cure local ailments, $12(12 \%)$ species are used for ritual and other purposes, $8(8 \%)$ species are used for rural fencin $\mathrm{g}$ and construction, $6(6 \%)$ species are used as spice and condiments, and atleast $4(4 \%)$ species are used in handicrafts (Figure 3). 


\begin{tabular}{|c|c|c|c|c|c|c|c|c|c|}
\hline 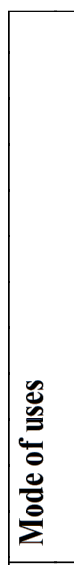 & 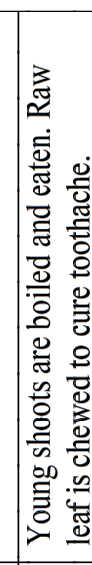 & 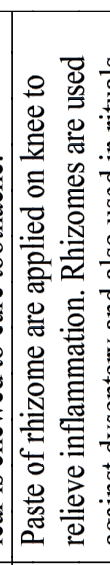 & & 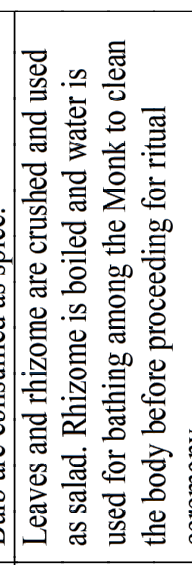 & 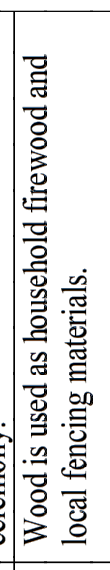 & 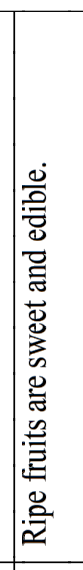 & 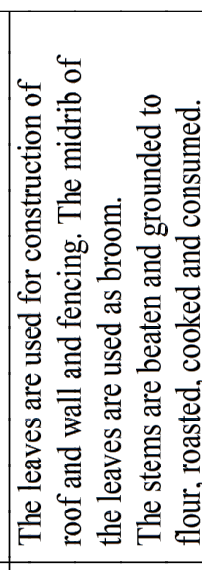 & 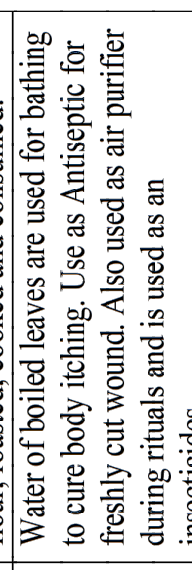 & 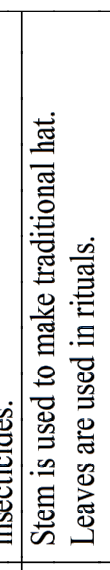 \\
\hline 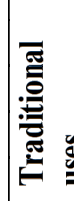 & 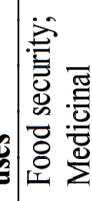 & 送 & & 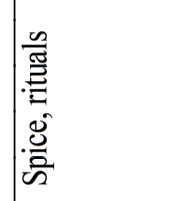 & 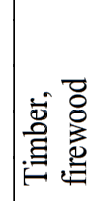 & 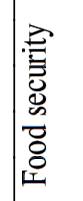 & 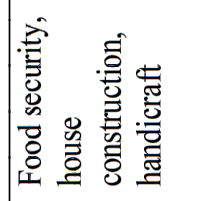 & 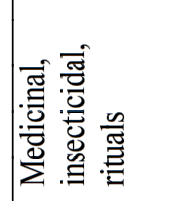 & 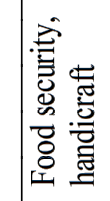 \\
\hline 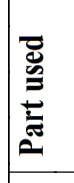 & 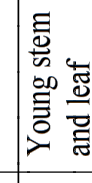 & 童 & 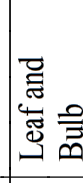 & & $\begin{array}{l}\overrightarrow{0} \\
\overline{0} \\
3\end{array}$ & 言 & 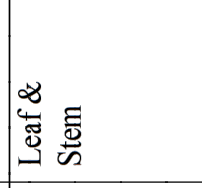 & 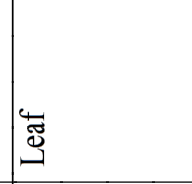 & 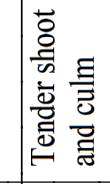 \\
\hline 部 & 目 & 客 & 8 & bे & 힝 & 宝 & 윰유 & 응 & ¿́ \\
\hline \begin{tabular}{|l} 
言 \\
产 \\
\end{tabular} & 䇏 & 䇏 & 产 & 景 & 导 & 潼 & 总 & 抟 & 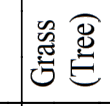 \\
\hline 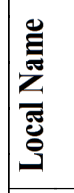 & 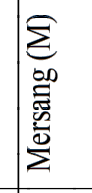 & 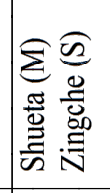 & 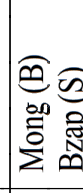 & 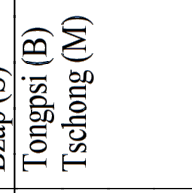 & 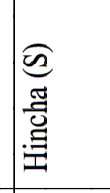 & $\begin{array}{l}\widehat{0} \\
\bar{d} \\
\bar{d} \\
\text { w }\end{array}$ & 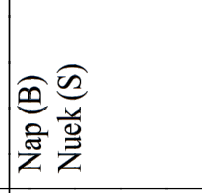 & 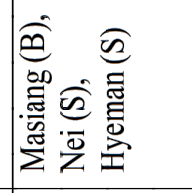 & 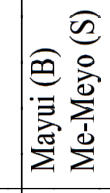 \\
\hline 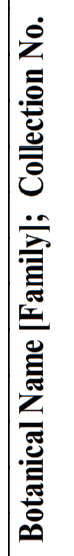 & 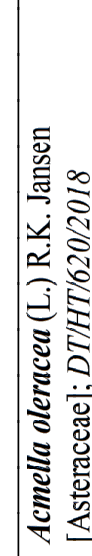 & 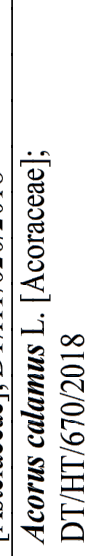 & 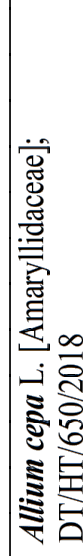 & 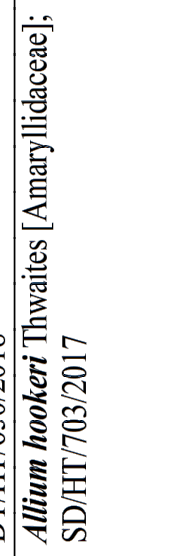 & 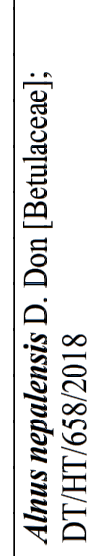 & 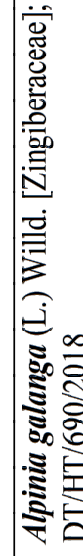 & 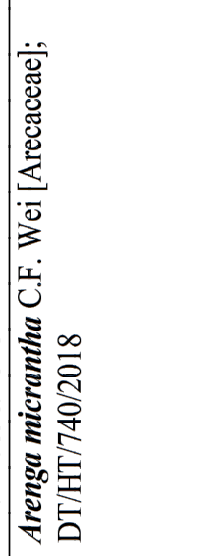 & 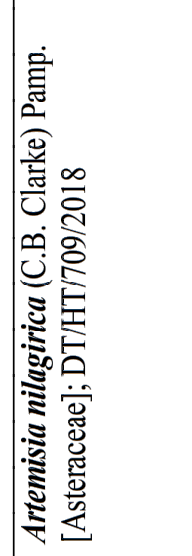 & 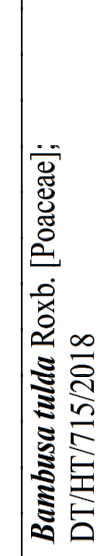 \\
\hline
\end{tabular}


Deki Tshering et al. 287

\begin{tabular}{|c|c|c|c|c|c|c|c|c|c|c|c|}
\hline 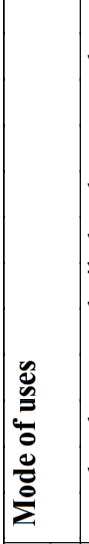 & 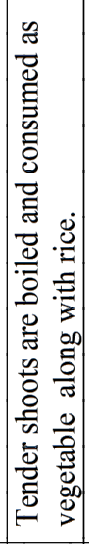 & 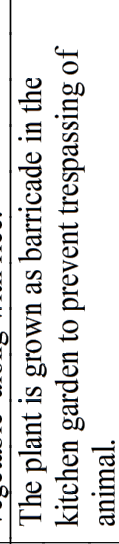 & 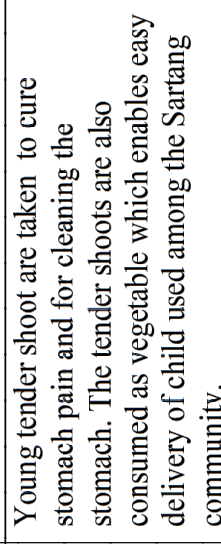 & 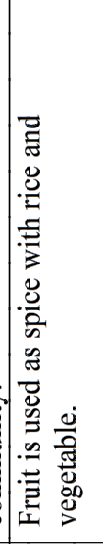 & 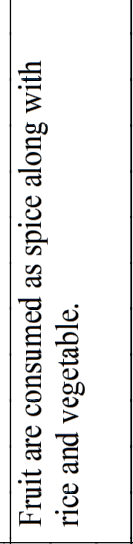 & 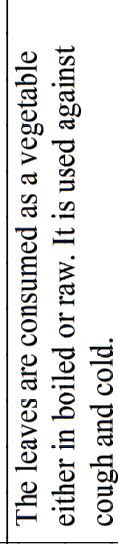 & 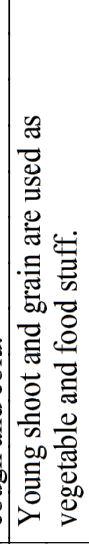 & 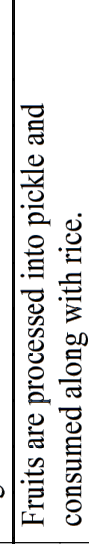 & 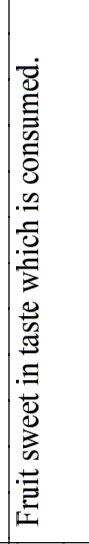 & 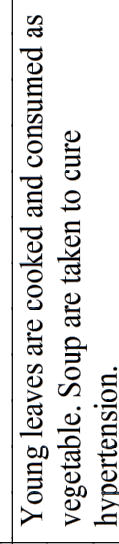 & 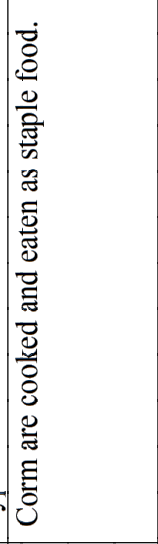 \\
\hline 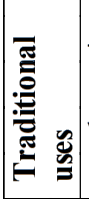 & 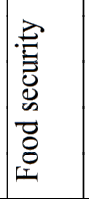 & 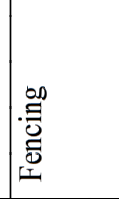 & 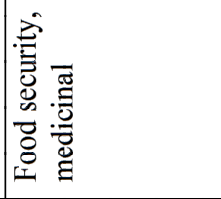 & 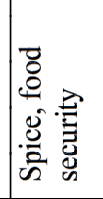 & 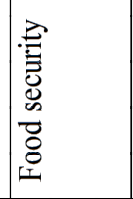 & 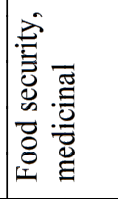 & 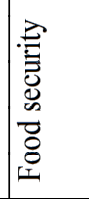 & 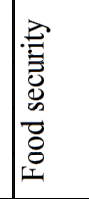 & 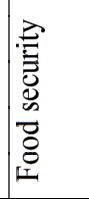 & 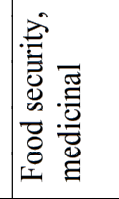 & 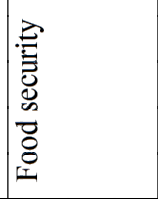 \\
\hline 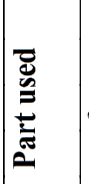 & 岕 & $\begin{array}{l}\frac{\overrightarrow{0}}{\frac{0}{2}} \\
\frac{0}{2} \\
\frac{0}{0} \\
\frac{0}{3}\end{array}$ & 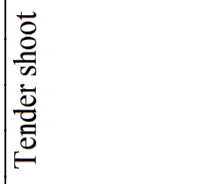 & 言 & 言 & 岕 & 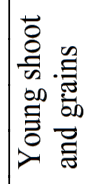 & 獄 & 壱 & 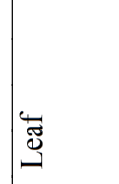 & 誈 \\
\hline 䒿 & 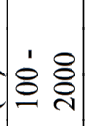 & 象 & ¿ి & ஓं & ¿́⿱亠凶禸 & ¿́⿱一் & ¿음 & 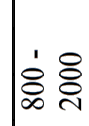 & ¿े & ச் & ¿̊ \\
\hline 音 & 竭 & 意 & 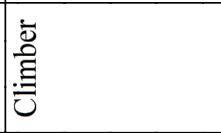 & 章 & 言 & $\underset{\Xi}{\Xi}$ & 言 & 衰 & 总 & 竞 & $\frac{0}{\underline{\underline{y}}}$ \\
\hline 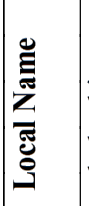 & 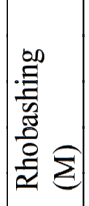 & 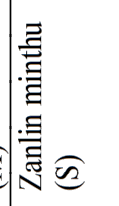 & 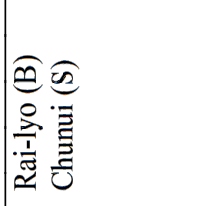 & 気 & 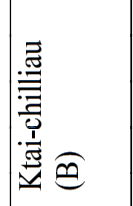 & 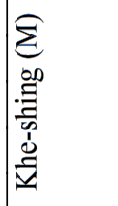 & 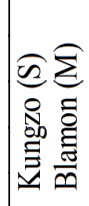 & 产 & 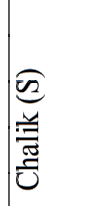 & 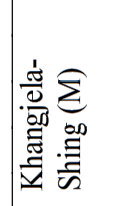 & 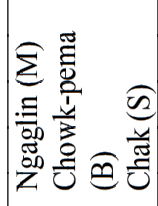 \\
\hline 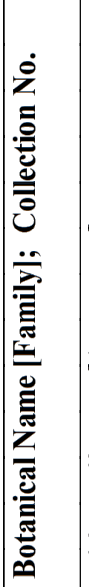 & 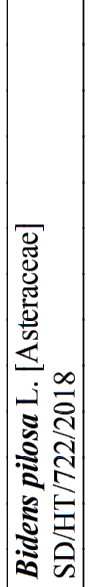 & 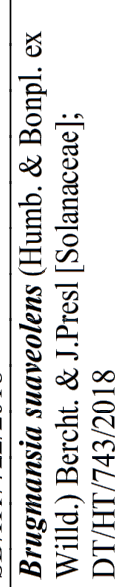 & 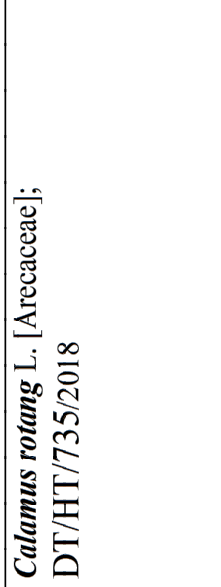 & 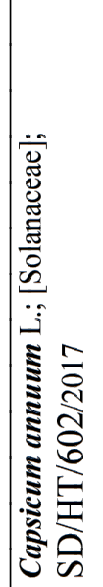 & 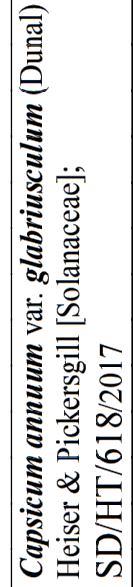 & 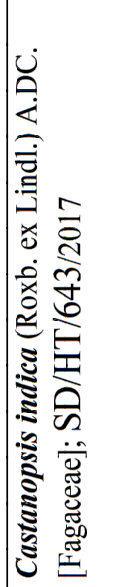 & 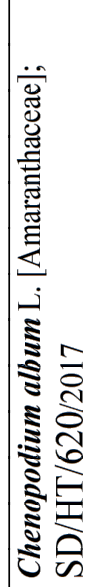 & 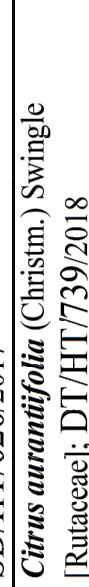 & 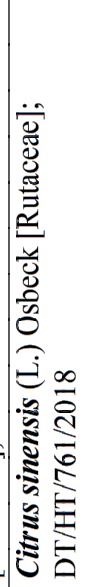 & 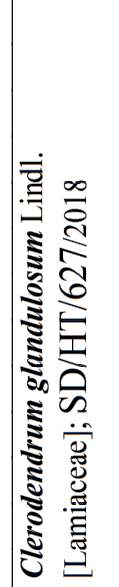 & 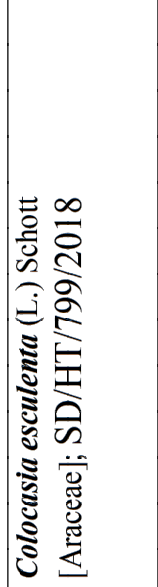 \\
\hline
\end{tabular}


288 Ethnobotanical appraisal of West Kameng and Tawang Sectors

\begin{tabular}{|c|c|c|c|c|c|c|c|c|c|c|c|c|c|c|}
\hline 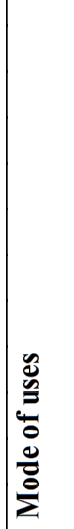 & 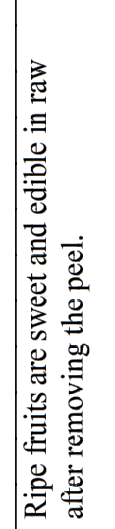 & 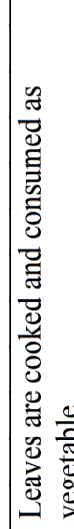 & 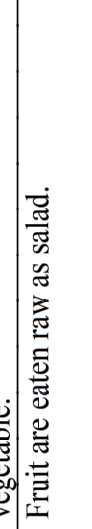 & 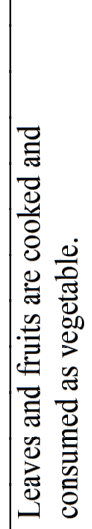 & 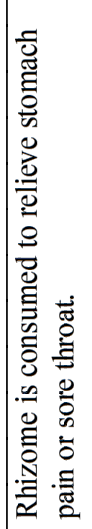 & 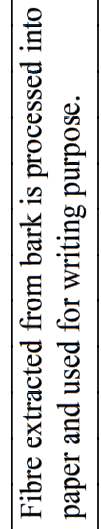 & 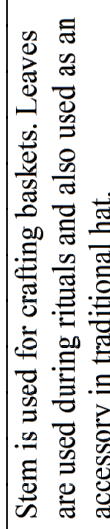 & 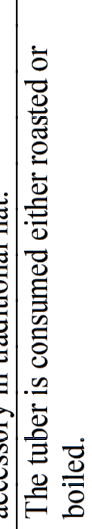 & 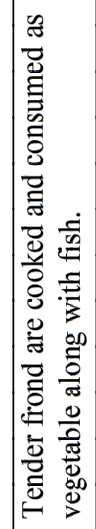 & 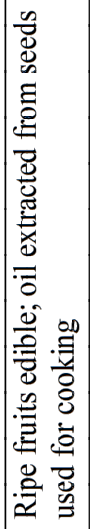 & 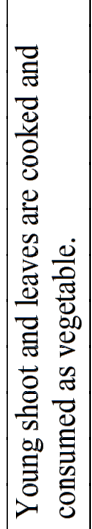 & 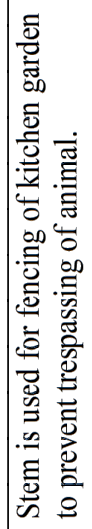 & 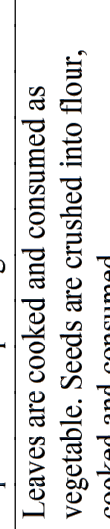 & 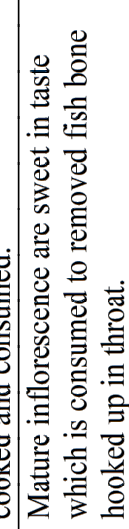 \\
\hline 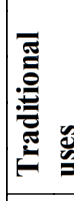 & 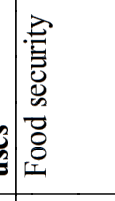 & 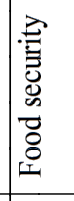 & 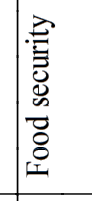 & 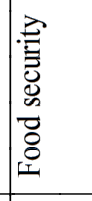 & 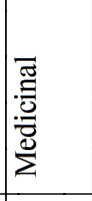 & 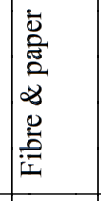 & 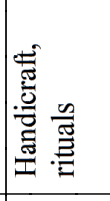 & 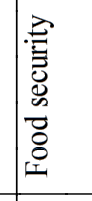 & 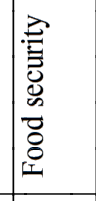 & 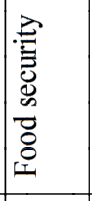 & 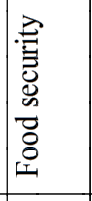 & 步 & 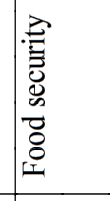 & 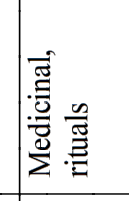 \\
\hline 离 & 言 & 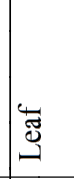 & 套 & 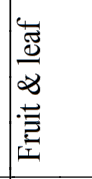 & 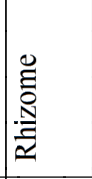 & 蓔 & 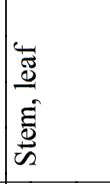 & 童 & 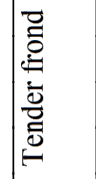 & 言 & 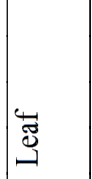 & 竞 & 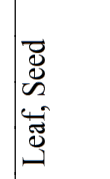 & 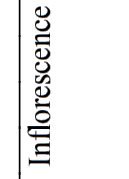 \\
\hline & 행요 & 家 & s. & 递 & ఏ் & 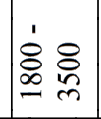 & 递 & 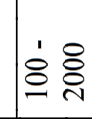 & 家 & 童 : & ఏ̊ & 임 & 递 & 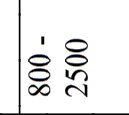 \\
\hline$\frac{6}{\sigma}$ & 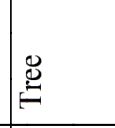 & 部 & 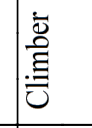 & 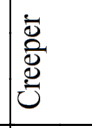 & 童 & 颜 & 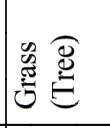 & 号 & $\frac{\underline{z}}{\underline{\underline{z}}}$ & 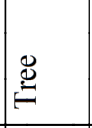 & 部 & 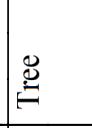 & 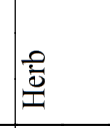 & 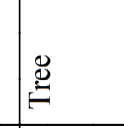 \\
\hline 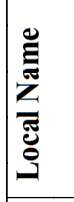 & 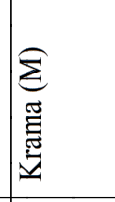 & 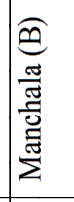 & 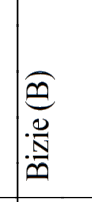 & 氕 & 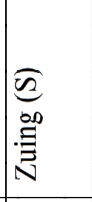 & 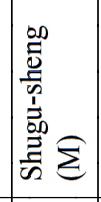 & 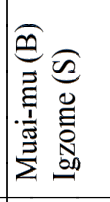 & 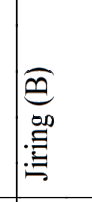 & 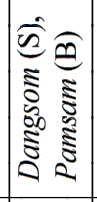 & 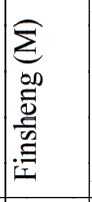 & 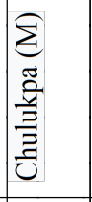 & 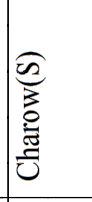 & 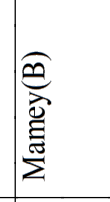 & 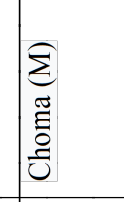 \\
\hline 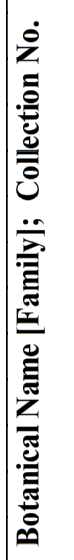 & 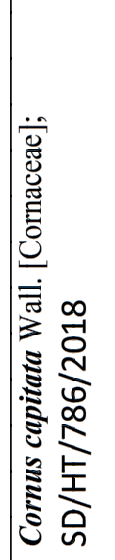 & 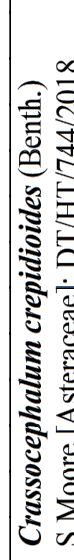 & 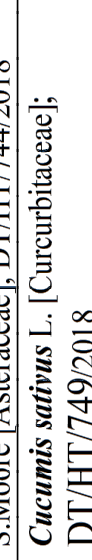 & 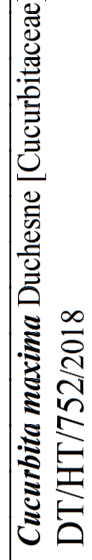 & 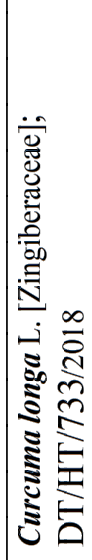 & 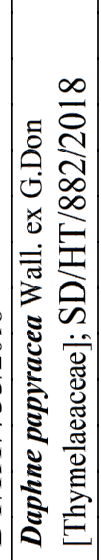 & 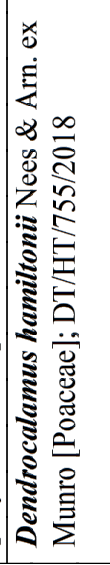 & 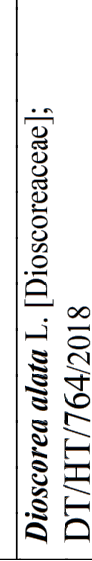 & 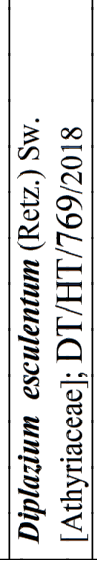 & 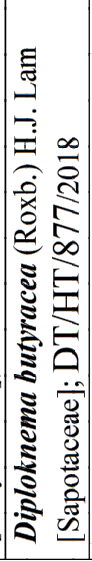 & 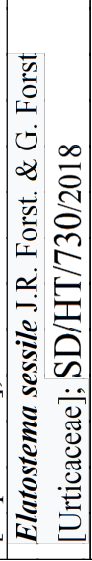 & 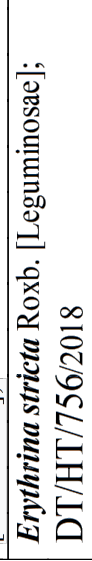 & 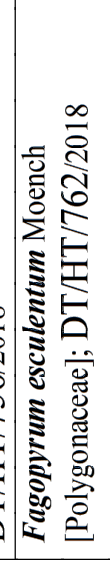 & 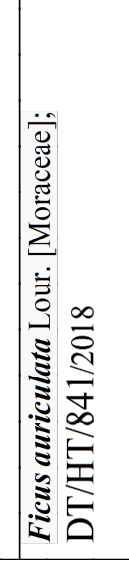 \\
\hline
\end{tabular}



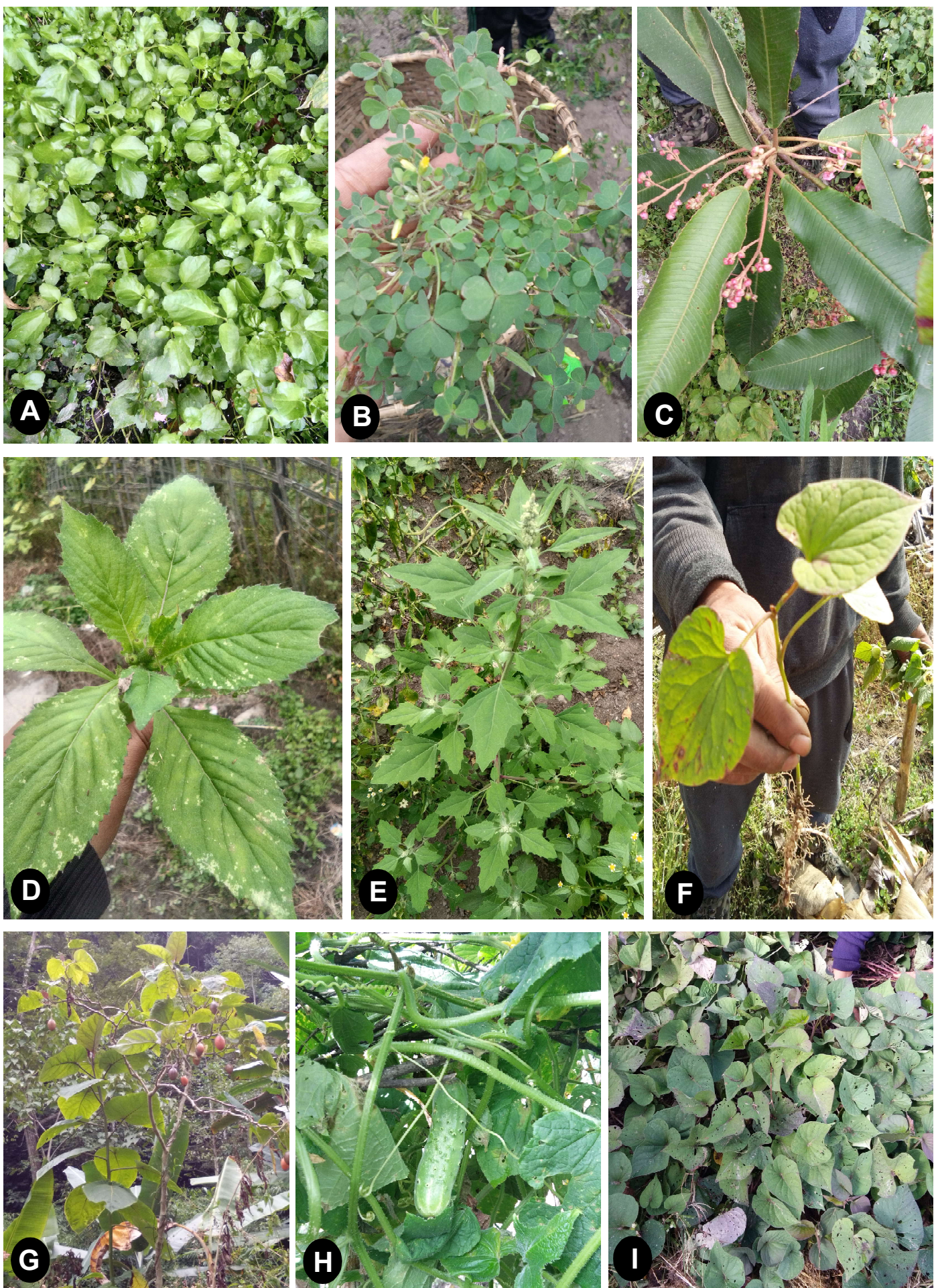

PLATE - I. Some ethnobotanical plants of Bugun, Sartang and Monpa biocultural landscape: A. Nasturtium officinale; B. Oxalis corniculata; C. Saurauia napaulensis; D. Crassocephalum crepidioides; E. Chenopodium album; F. Houttuynia cordata; G. Solanum betaceum; H. Cucumis sativa; I. Ipomoea batatas 
290 Ethnobotanical appraisal of West Kameng and Tawang Sectors

\begin{tabular}{|c|c|c|c|c|c|c|c|c|c|c|c|c|}
\hline 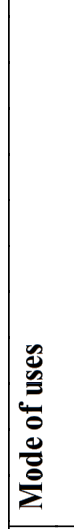 & 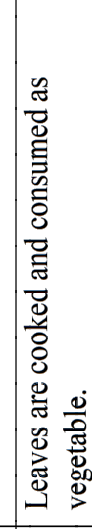 & 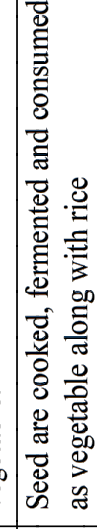 & 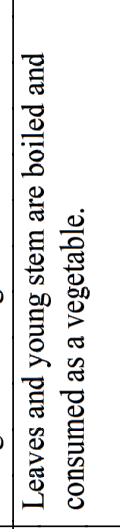 & 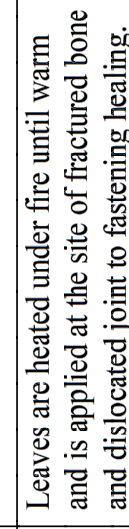 & 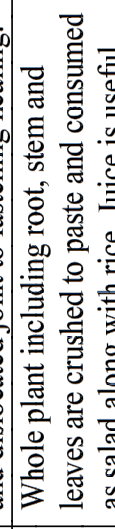 & 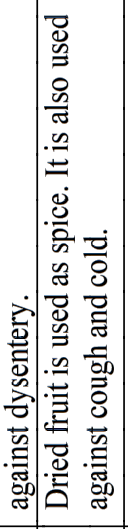 & 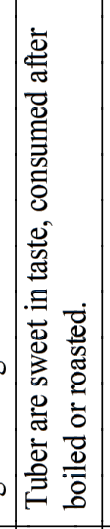 & 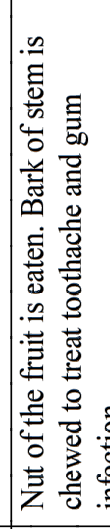 & 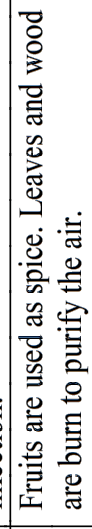 & 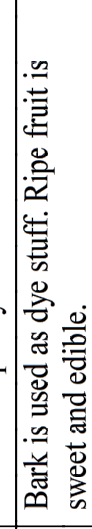 & 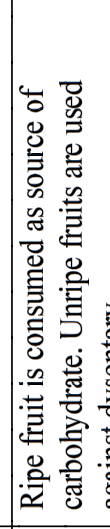 & 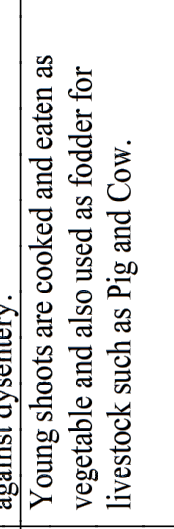 \\
\hline 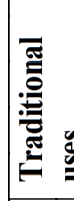 & 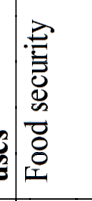 & 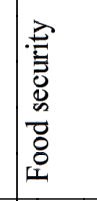 & 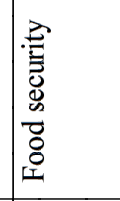 & 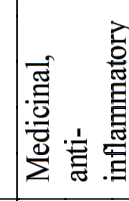 & 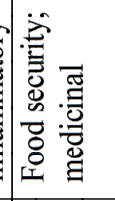 & 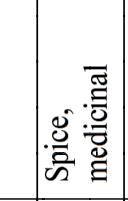 & 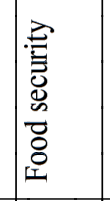 & 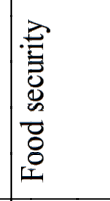 & 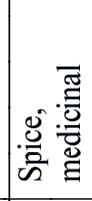 & 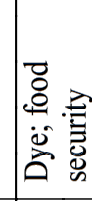 & 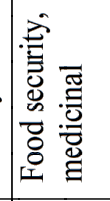 & 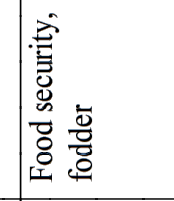 \\
\hline 总 & 容 & \begin{tabular}{|l}
$\overrightarrow{8}$ \\
on
\end{tabular} & 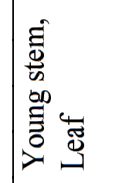 & 岁 & 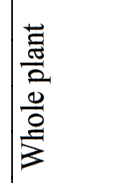 & 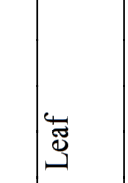 & 离 & 害 & 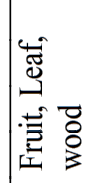 & 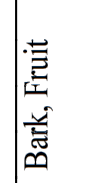 & 言 & 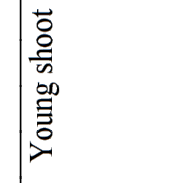 \\
\hline $\bar{Z}$ & ¿̀ & 字商 & 官各 & 음 & 웅 & : & 家 & 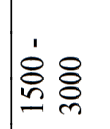 & ذ̇ & 윰 & ذి & 宫 \\
\hline 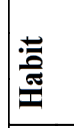 & 噫 & 或 & 咅 & 蛮 & 䇏 & 总 & 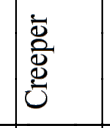 & 兽 & 总 & 䞶 & $\frac{\underline{\underline{z}}}{\underline{\underline{x}}}$ & 讋 \\
\hline $\begin{array}{l}\frac{\mathrm{E}}{\mathrm{E}} \\
\frac{\mathrm{E}}{\mathrm{E}}\end{array}$ & 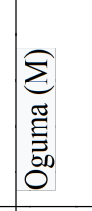 & 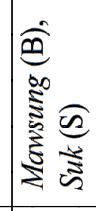 & 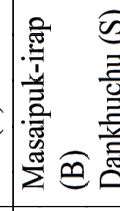 & 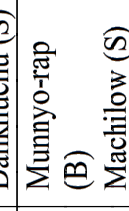 & 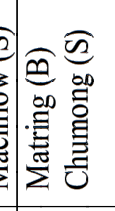 & $\sum_{\frac{\pi}{3}}$ & 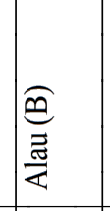 & 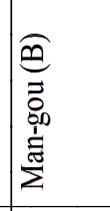 & 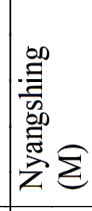 & 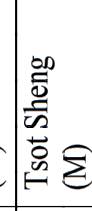 & 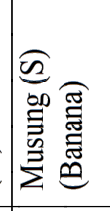 & 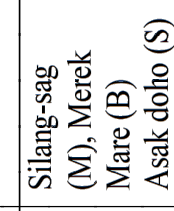 \\
\hline 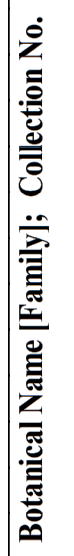 & 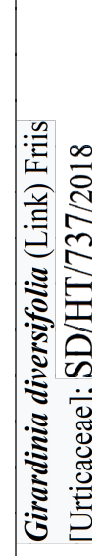 & 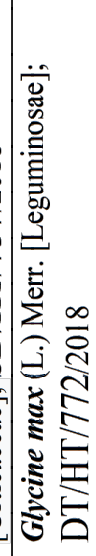 & 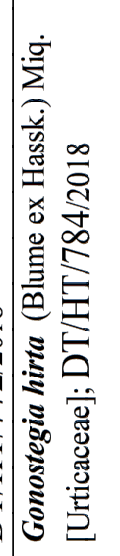 & 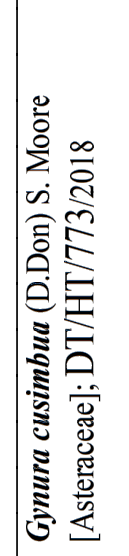 & 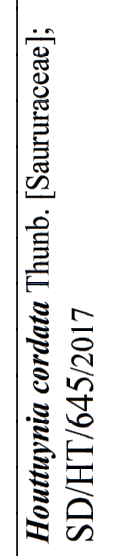 & 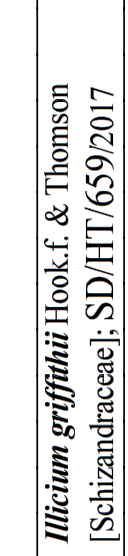 & 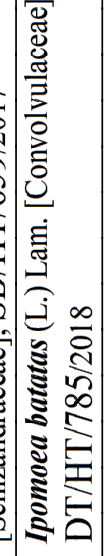 & 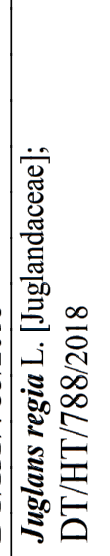 & 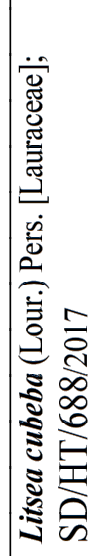 & 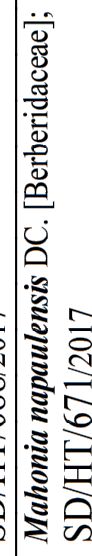 & 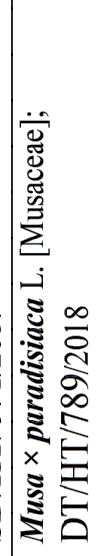 & 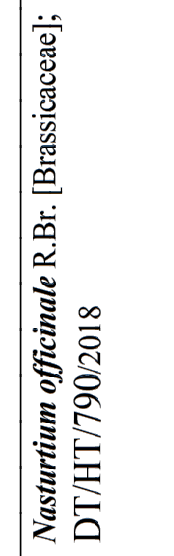 \\
\hline
\end{tabular}


Deki Tshering et al. 291

\begin{tabular}{|c|c|c|c|c|c|c|c|c|c|c|c|c|c|c|}
\hline & 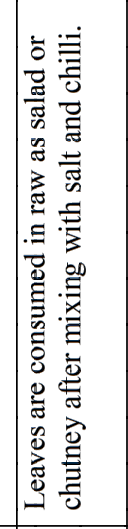 & 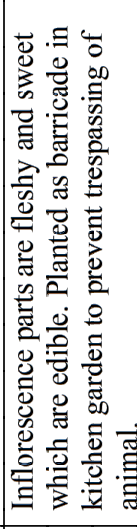 & 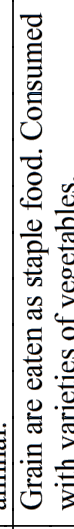 & 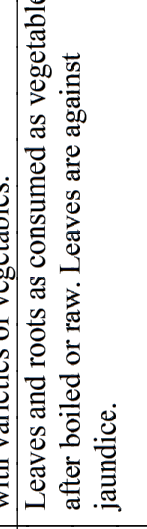 & 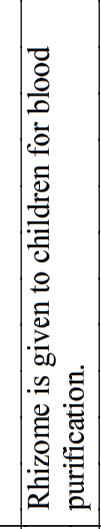 & 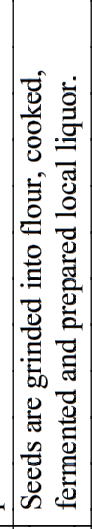 & 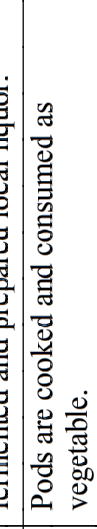 & 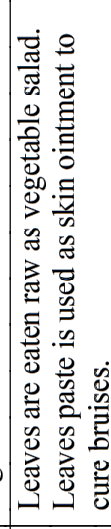 & 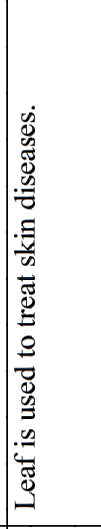 & 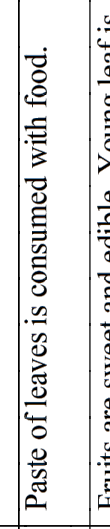 & 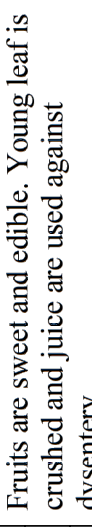 & 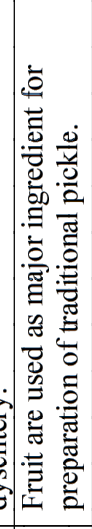 & 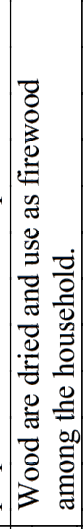 & 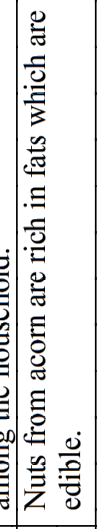 \\
\hline $\bar{E}$ & 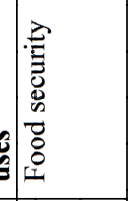 & 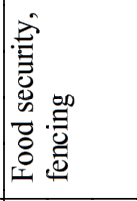 & 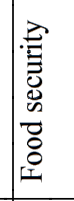 & 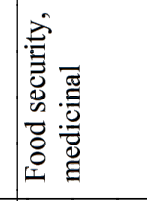 & 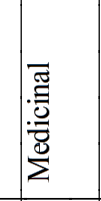 & 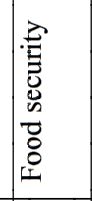 & 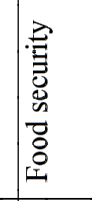 & 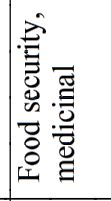 & 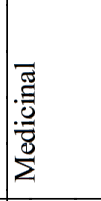 & 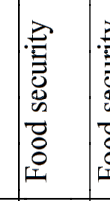 & 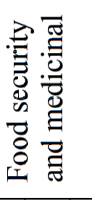 & 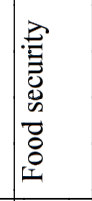 & & 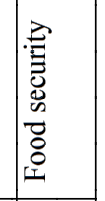 \\
\hline f & 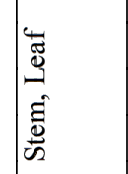 & 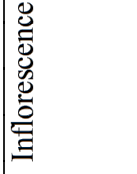 & हू हू है & 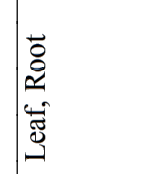 & 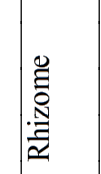 & 怘 & : & 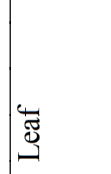 & $\Phi$ & 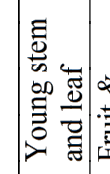 & & 搳 & $\mid$\begin{tabular}{|l}
$\overrightarrow{0}$ \\
0 \\
3 \\
3
\end{tabular} & 言 \\
\hline & 宓 & ¿̊ & ¿ి. & 准 & 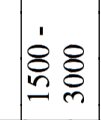 & 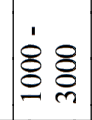 & 主学 & 完 & 定要 & 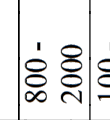 & 家戹 & 宓 & : & 证: \\
\hline & 竧 & 旗 & 氰 & 避 & 童 & 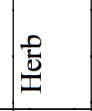 & $\begin{array}{l}\text { 递 } \\
\\
\end{array}$ & 童 & 递 & 总 & $\stackrel{\mathscr{Z}}{\sharp}$ & : & 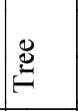 & : \\
\hline 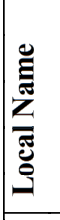 & 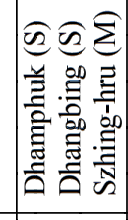 & & $\begin{array}{l}\widehat{\tilde{n}} \\
\bar{z}\end{array}$ & 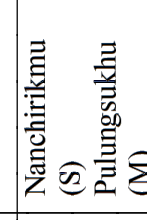 & 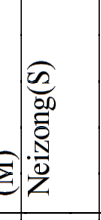 & $\begin{array}{l}\widehat{\hat{\theta}} \\
\underline{z} \\
\overline{0} \\
\tilde{d}\end{array}$ & 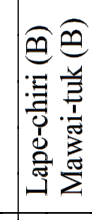 & 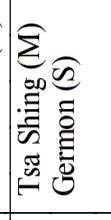 & 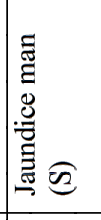 & 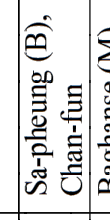 & & 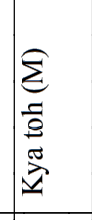 & 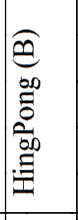 & 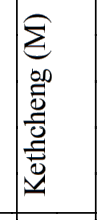 \\
\hline 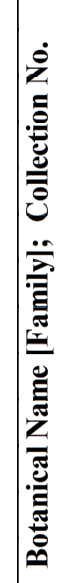 & 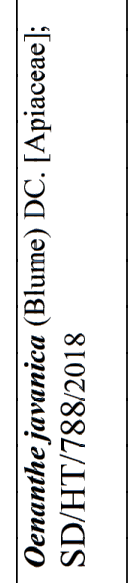 & 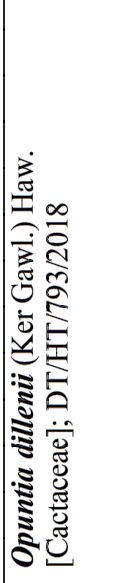 & 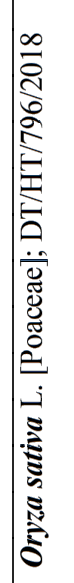 & 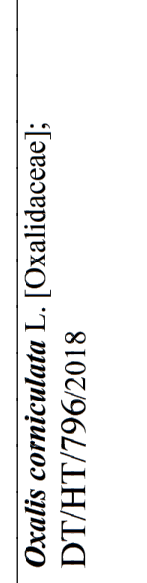 & 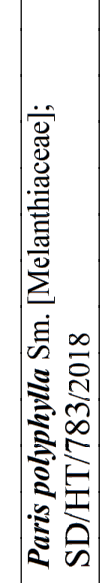 & 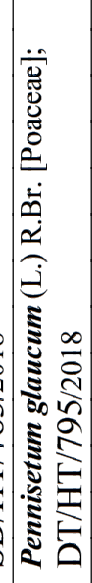 & 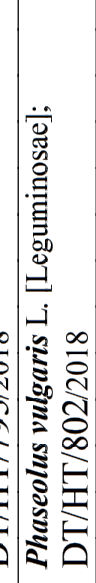 & 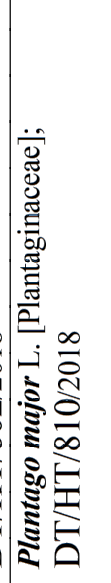 & 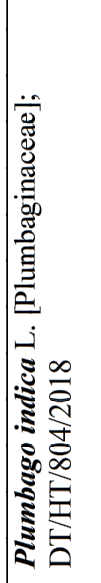 & 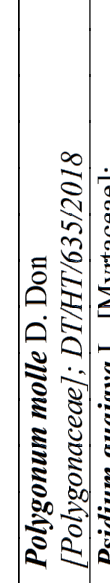 & 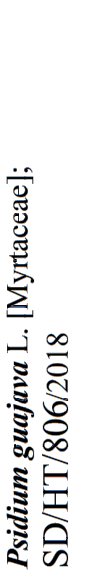 & 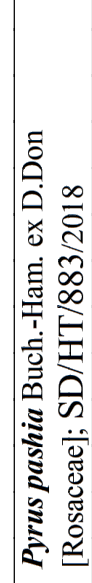 & 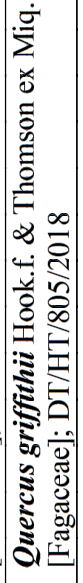 & 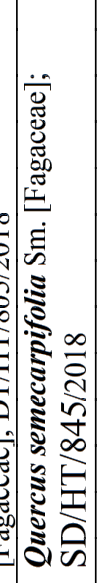 \\
\hline
\end{tabular}




\begin{tabular}{|c|c|c|c|c|c|c|c|c|c|c|c|c|}
\hline 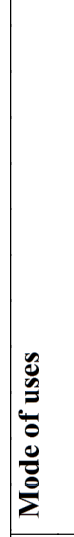 & 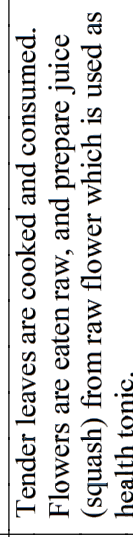 & 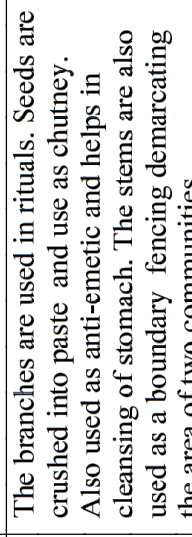 & 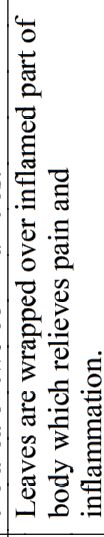 & 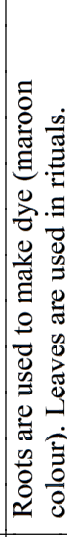 & 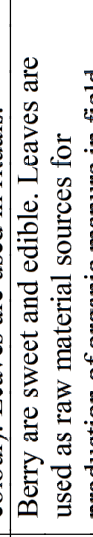 & 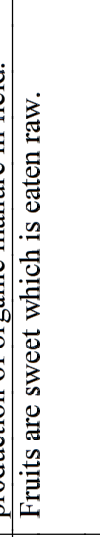 & 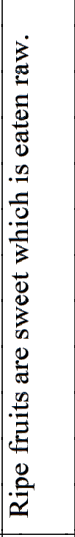 & 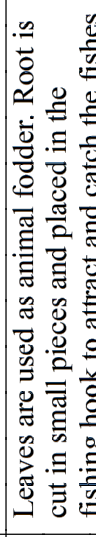 & 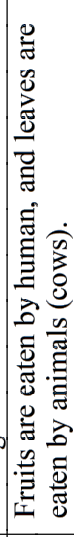 & 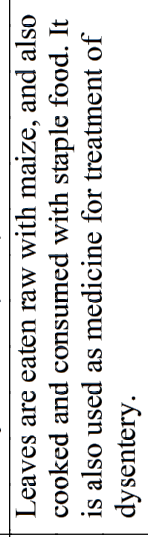 & 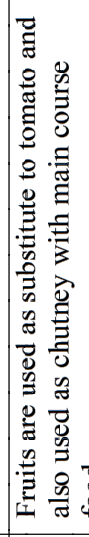 & 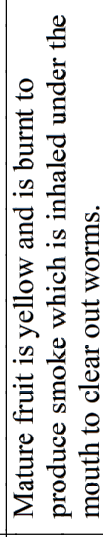 \\
\hline 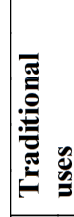 & $\frac{n}{\underline{n}}$ & 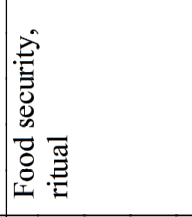 & 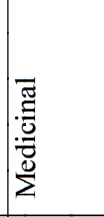 & 齿 & 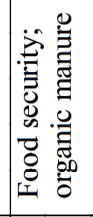 & 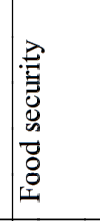 & 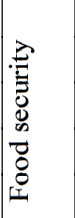 & 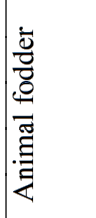 & 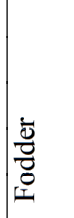 & 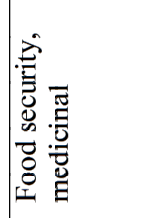 & 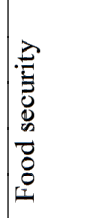 & 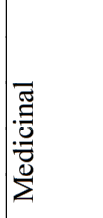 \\
\hline 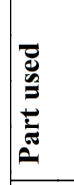 & 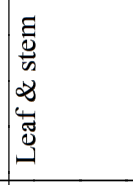 & 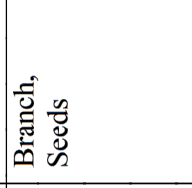 & 岕 & 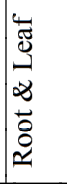 & 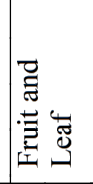 & 莹 & 嗀 & 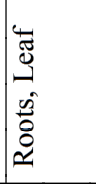 & 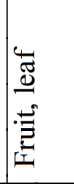 & 岕 & 壱 & 言 \\
\hline 䒿 & : & 용 & 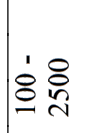 & 客 & 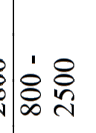 & 官号 & : & 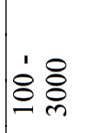 & 음 & ¿̊ & 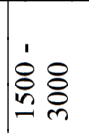 & 它 \\
\hline נِّ & 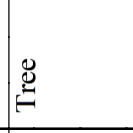 & 惫 & $\frac{\vec{E}}{\frac{E}{2}}$ & 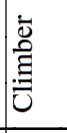 & 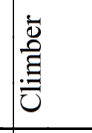 & 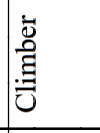 & 产 & 童 & $\underset{\mathscr{Z}}{\mathscr{Z}}$ & 童 & 差 & 咅 \\
\hline 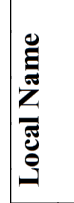 & 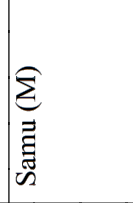 & 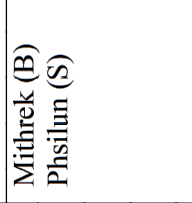 & 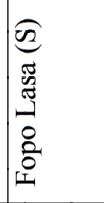 & 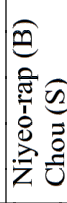 & 竞 & 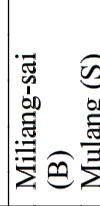 & 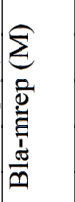 & 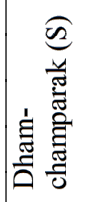 & 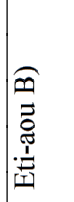 & 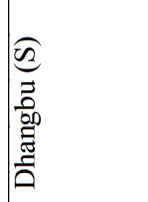 & 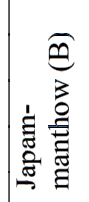 & 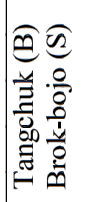 \\
\hline 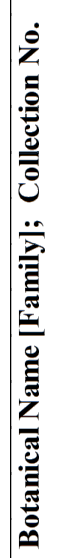 & 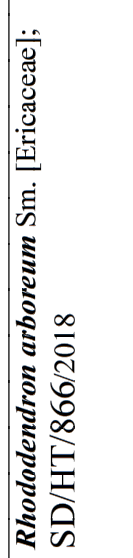 & 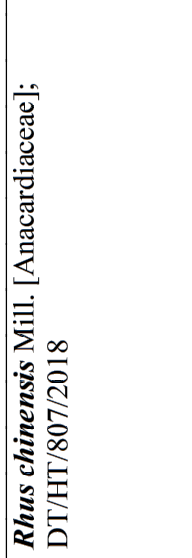 & 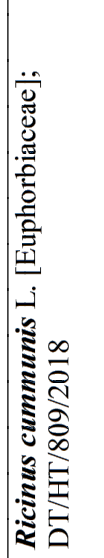 & 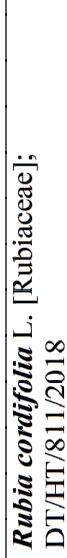 & 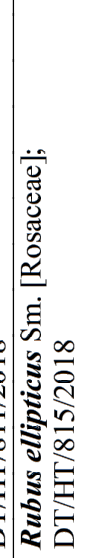 & 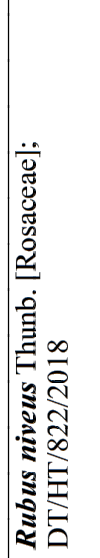 & 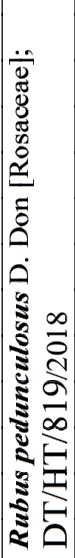 & 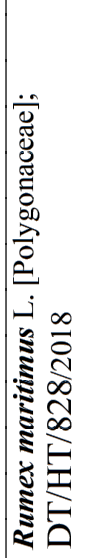 & 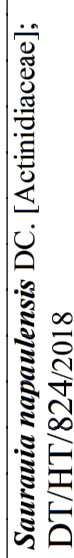 & 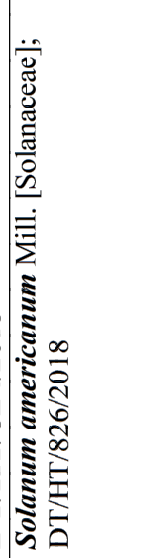 & 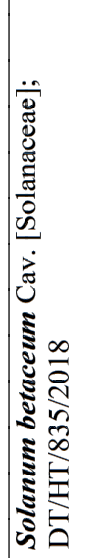 & 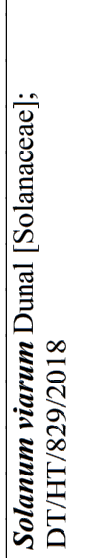 \\
\hline
\end{tabular}




\begin{tabular}{|c|c|c|c|c|c|}
\hline 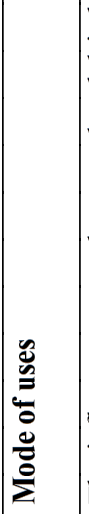 & 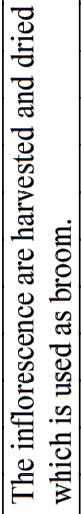 & 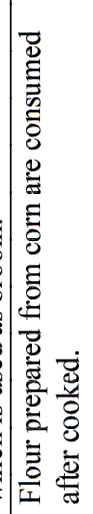 & 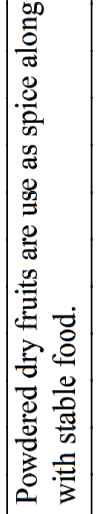 & 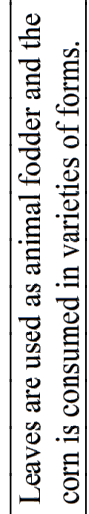 & 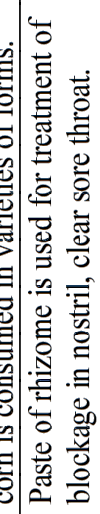 \\
\hline 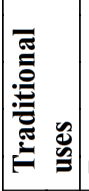 & छี & 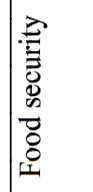 & 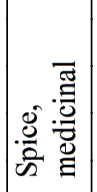 & 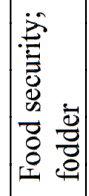 & 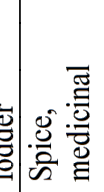 \\
\hline 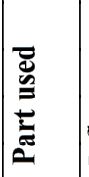 & 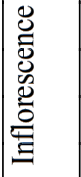 & 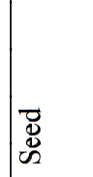 & 军 & 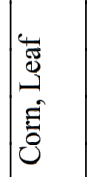 & 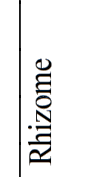 \\
\hline 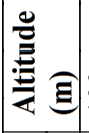 & ¿ & 竎 & ○ ڤ & 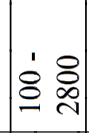 & ț \\
\hline 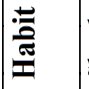 & 总 & 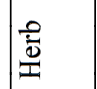 & 苋 & 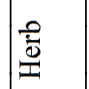 & 产 \\
\hline 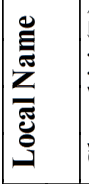 & 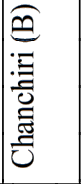 & 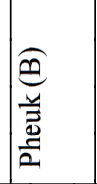 & 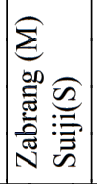 & 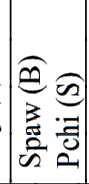 & 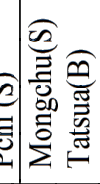 \\
\hline 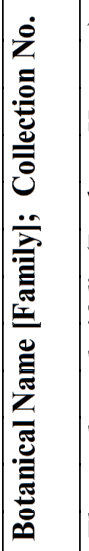 & 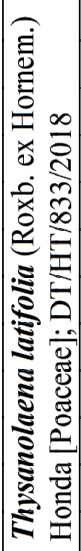 & 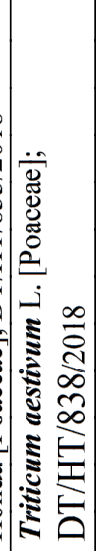 & 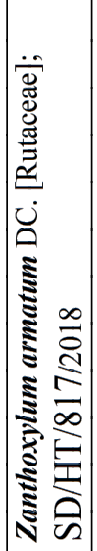 & 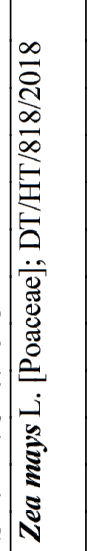 & 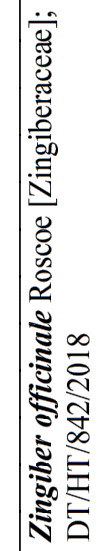 \\
\hline
\end{tabular}

\section{Harvesting of plant parts}

Almost 33 (41\%) of the species reported from BugunSartang-Monpa biocultural landscape are harvested from young shoot and leafy part, $31(38 \%)$ species are harvested from reproductive parts which include inflorescence, flowers, fruits and seeds, $10(12 \%)$ species are harvested from stem parts which include wood and bark, whereas only $7(9 \%)$ species are harvested from underground plant parts which include rhizome, roots, tubers, corm and bulb (Figure 4).

\section{Ecological status}

Majority of 76 (61\%) of ethnobotanical species reported from target biocultural landscape are found growing in subtropical and temperate region with altitude ranging from $1000-2500 \mathrm{~m}$ AMSL, 40 (32\%) species are adapted to tropical and subtropical region with altitude ranging from $100-2500 \mathrm{~m}$ from mean sea level whereas only $9(7 \%)$ of the total species reported are capable of adapting to wide range of climatic zone ranging from both tropical and temperate region with altitude $100-$ $3500 \mathrm{~m}$. Of the total 77 species, 55 (71\%) species are harvested from wild sources which include wild food and medicinal plants, rituals, handicrafts and other plants while $22(29 \%)$ species harvested are from cultivated sources which include staple food plants such as rice, millets, Maize, Beans, Cucumber, Pumpkin, vegetable species (Figure 5).

\section{Diversity of wild food plants}

Present investigation has revealed that the Bugun, Sartang and Monpa biocultural landscape of West Kameng and Tawang district uses good number of wild edible plants which are collected from their community conserved forest area. Some of the commercially viable and economically significant wild food plant species found in the region are: Arenga micrantha, Castanopsis indica, Clerodendrum glandulosum, Diplocknema butyracea, Elatostema sessile, Gonostegia hirta, Houttuynia cordata, Juglans regia, Quercus semecarpifolia, Quercus griffithii, Rhododendron arboreum, and Zanthoxylum armatum. Conservation of these wild edible species are usually done in the community forest land and sacred grooves.

\section{Cultivated plants of economic and cultural significance}

Statistics of ecological status revealed that $22(29 \%)$ species harvested from cultivated sources are mostly 


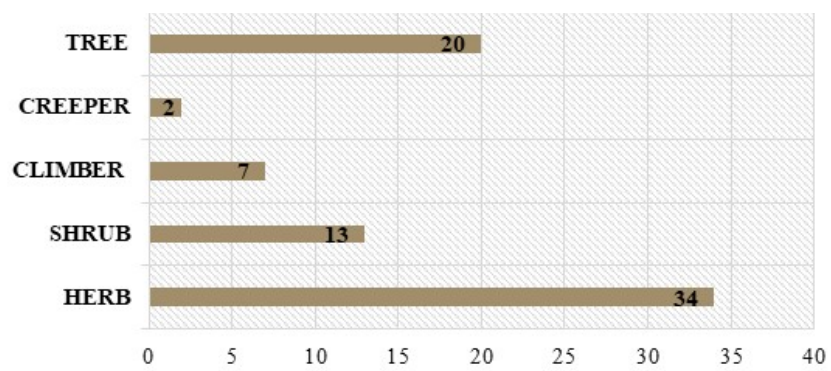

Figure 2. Habit status of ethnobotanical plants of Bugun, Sartang and Monpa biocultural landscape

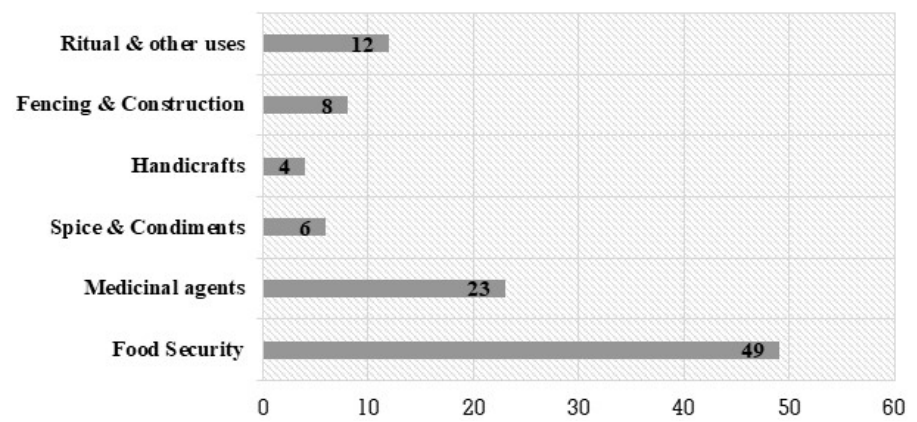

Figure 3. Diverse uses of ethnobotanical species of Bugun, Sartang and Monpa biocultural landscape

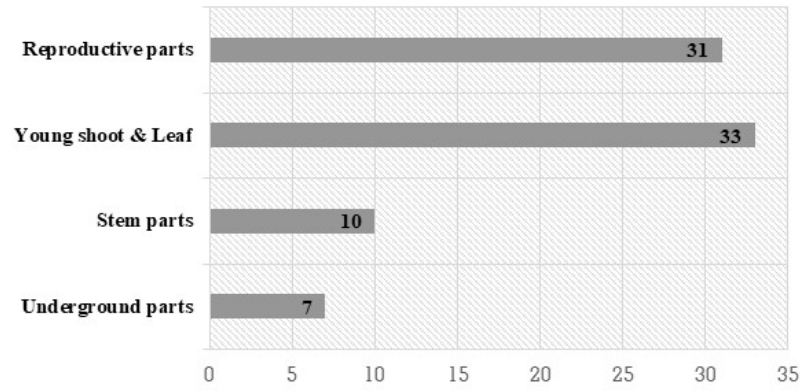

Figure 4. Statistics of plant parts harvested from Bugun, Sartang and Monpa biocultural landscape

stable food plants such as Cucumis sativa, Curcurbita maxima, Chenopodium album, Glycine max, Oryza sativa, Pennisetum macrourum, Phaseolus vulgaris, Solanum betaceum, Zea mays, and other vegetable species. These staple food and vegetable plants are economically and commercially viable species which has the potential to sustain rural economy and the livelihood. Some of the cultivated plants such as rice and maize have the close cultural linkages with the target local communities (Figure 6).

\section{Plant used in traditional folk medicine, rituals and other purposes}

Of the 77 species reported, 23 (22\%) of the species are invariably used in traditional folk medicines. The medicinal plants of economic and commercial interest found in the Bugun, Sartang and Monpa biocultural landscape are: Acmella oleracea, Polygonum molle, Acorus calamus, Allium hookeri, Alpinia galanga, Artemisia nilagirica, Curcuma longa, 


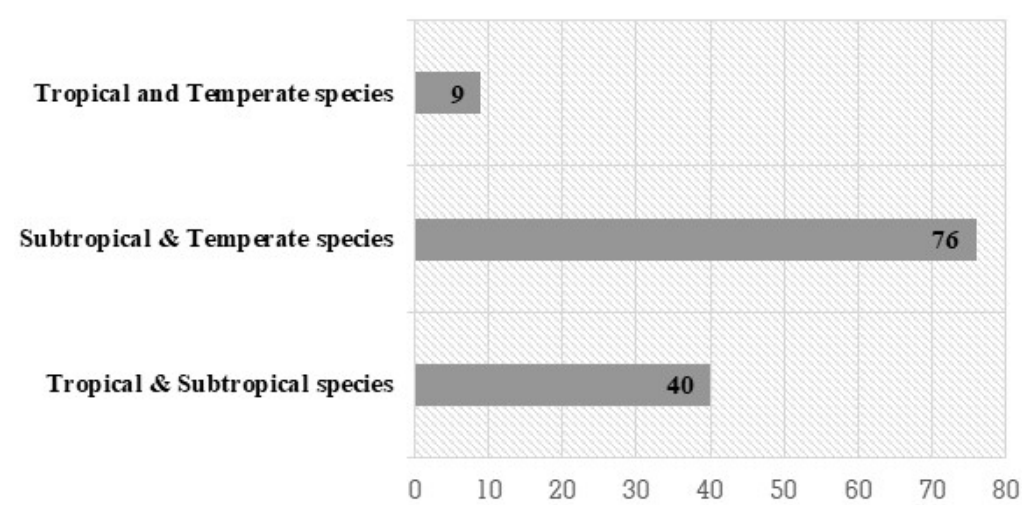

Figure 5. Distribution and altitudinal range of ethnobotanical plants of Bugun, Sartang and Monpa biocultural landscape

Houttuynia cordata, Illicium griffithii, Litsea cubeba, Paris polyphylla, Plumbago indica, Rubia cordifolia, Ricinus cummunis, and Zingiber officinale. These medicinal plants are easily available in their eco-cultural landscape which are usually nurtured by the community.

Some of the plant species used in rituals, spice and condiments, handicrafts, fencing and construction are: Allium hookeri, Alnus nepalensis, Bambusa tulda, Brugmansia suaveolens, Daphne papyracea, Dendrocalmus hamiltonii, Ficus auriculata, Rhododendron arboreum, Rhus chinensis, and Rubia cordifolia.

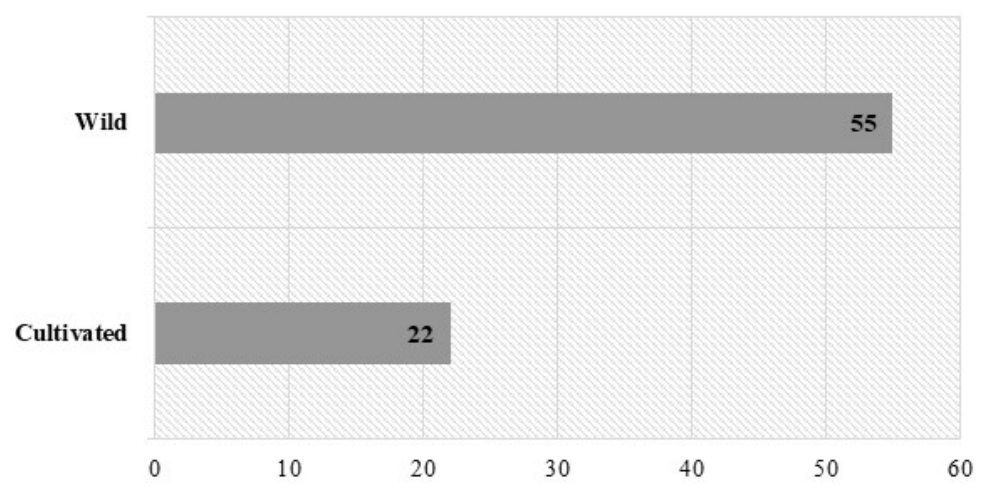

Figure 6. Cultivation status of ethnobotanical plants of Bugun, Sartang and Monpa biocultural landscape

\section{Species potential to ensure rural livelihood security}

The species which are commonly found and frequently used by the local residents having potential to ensure rural livelihood security to the target communities and capable of transforming the rural economy by augmenting trade and commerce activities in the target biocultural landscape are: Alnus nepalensis, Allium hookeri, Acorus calamus, Bambusa tulda, Clerodendrum glandulosum, Daphne papyracea, Elatostema sessile, Ficus auriculata, Glycine max, Gonostegia hirta, Illicium griffithii, Ipomoea batatas, Juglans regia, Litsea cubeba, Paris polyphylla, Quercus griffithii, Qercus semecarpifolia, Rhododendron arboreum, Rubia cordifolia, Zanthoxylum armatum and Zea mays. 


\section{CONCLUSION}

It is concluded that the lesser known tribe Bugun and Sartang have their rich traditional knowledge related to diverse utilization of ethnobotanical resources of their biocultural landscape. The species found in their biocultural landscape are closely related to their local faith and belief system, and livelihood support system of the target communities. The Monpa community which are economically much better in the same biocultural landscape also have the strong linkages with plant resources. The food, medicinal and cultural plants used by the three culturally distinct and heterogeneous communities have deep rooted cultural connotations which are directly linked with economy, livelihood, faith and belief system of the communities. Although majority species are harvested from the wild sources but these species are also capable of adapting to wide range of agro-climatic zone ranging from tropical to temperate zones. The most commonly and frequently harvested species have the potential to boost rural economy, trade and commerce in the region. Such potential species should be encouraged for conservation in community forest land, and should be brought under cultivation trial in different agro-climatic regions to enhance productivity and to ensure rural livelihood security of economically marginalised and lesser known communities living in the target biocultural landscape.

\section{Acknowledgements}

The first and last authors are thankful to DBT, GoI for financial support to last author (HT) through DBT Twinning Project and also thankful to UGC New Delhi for funding support through CPEB-II project. The second and third authors are thankful to GBPNIHESD Almora, MoEF GoI for funding support under IERP programme.

\section{LITERATURE CITED}

Anonymous, 2011. CENSUS report: Arunachal Pradesh. Ministry of Home Affairs, Government of India. Pp. 5 - 20.

Hooker, J.D. 1872-1897. The Flora of British India, Vols. 1-7. L. Reeve \& Co Ltd, Ashford, Kent. London.

Jain, S.K. \& Rao, R.R.1977. A Handbook of Field and Herbarium Methods. Today \& Tomorrow's Printers and Publishers, New Delhi.

Kanjilal, U.N.; Kanjilal, P.C; Das. A. \& Purkaystha, C. 1934. Flora of Assam, Vol. 1, Assam Govt. Press, Shillong.

Kanjilal, U.N.; Kanjilal, P.C. \& Das, A. 1938. Flora of Assam, Vol. 2, Assam Govt. Press, Shillong.

Kanjilal, U.N.; Kanjilal, P.C.; Das. A. \& Dey, R.N. 1939. Flora of Assam, Vol. 3, Assam Govt. Govt. Press, Shillong.

Kanjilal, U.N.; Kanjilal, P.C; Das, A, \& Dey, R.N. 1940. Flora of Assam, Vol. 4, Assam Govt. Govt. Press, Shillong.

Martin, G.J. 2008. Ethnobotany: A Methods Manual, People and Plants Conservation Series. Earthscan, UK and USA. Pp. $10-160$.

Mazhar, F; Buckles, D; Satheesh, P.V \& Akhter F. 2007. Food Sovereignty and Uncultivated Biodiversity in South Asia. Academic Foundation, New Delhi. Pp. 5-20.

Norbu, T. 2008. The Monpas of Tawang: Arunachal Pradesh. Director of Research, Department of Cultural Affairs, Arunachal Pradesh. Pp. 40 - 180. 
Srivastava, R,C; Singh, R.K; Mukherjee, T.K. 2010. Indigenous biodiversity of Apatani tribe of Arunachal Pradesh for sustainable livelihoods. Indian Journal of Traditional Knowledge 9 (3): 432 - 442.

Tag, H; Tsering, J; Hui, P.K; Gogoi, B.J \& Veer V. 2014. Nutritional potential and traditional uses of high altitude wild edible plants in Eastern Himalayas, India, WASET, 8 (2): 395 -400 .

Tsering J; Gogoi B.J; Hui, P.K; Tam, N \& Tag, H. 2017. Ethnobotanical appraisal on wild edible plants used by the Monpa community of Arunachal Pradesh. Indian Journal of Traditional Knowledge 16 (4): 626 - 637.

Tsering, J \& Tag, H. 2015. High altitude ethnomedicinal plants of Western Arunachal Himalayan Landscape, Pleione 9 (1): 18 - 25.

Tsering, J; Tag, H; Gogoi, B.J \& Veer, V. 2016. Traditional anti-poison plants used by the Monpa tribe of Arunachal Pradesh, In: Herbal insecticides, repellents and biomedicines: Effectiveness and commercialization, edited by $\mathrm{V}$ Veer \& $\mathrm{R}$ Gopalakrishnan, (Springer India). Pp. 189 - 203.

www.theplantlist.org

www.efloras.org 